\title{
Suppressive effect of microRNA-126 on oral squamous cell carcinoma in vitro
}

\author{
XIAOLUN YANG, HANJIANG WU and TIANYOU LING
}

Department of Stomatology, The Second Xiangya Hospital of Central South University, Changsha, Hunan 410011, P.R. China

Received September 5, 2013; Accepted March 6, 2014

DOI: $10.3892 / \mathrm{mmr} .2014 .2171$

\begin{abstract}
MicroRNA-126 (miR-126), an endothelial-specific miRNA located within intron 7 of epidermal growth factor-like domain 7 (EGFL7), has been demonstrated to act as a tumor suppressor in various types of human cancer. However, its role in oral squamous cell carcinoma (OSCC) remains unclear. In the present study, we revealed that the expression of miR-126 was significantly decreased in OSCC tissues, when compared with that in their matched adjacent tissues, and its expression level was also reduced in Tca8113, OSCC-15 and CAL27 cell lines compared with normal tissues. The protein expression of EGFL7 was upregulated in OSCC tissues compared with their matched adjacent tissues as well as normal tissues, and Tca8113, OSCC-15 and CAL27 cells additionally demonstrated a positive expression of EGFL7. The overexpression of miR-126 significantly reduced the protein expression of EGFL7 in OSCC-15 cells, while transfection with the miR-126 inhibitor upregulated the EGFL7 protein level in OSCC-15 cells. Furthermore, transfection with an miR-126 mimic into OSCC-15 cells markedly suppressed cell proliferation, cell cycle progression, cell invasion and colony formation, while inducing cell apoptosis, which contrasted with the effects of transfection with an miR-126 inhibitor. The overexpression of miR-126 suppressed the secretion of two key regulators of angiogenesis, vascular endothelial growth factor (VEGF) and basic fibroblast growth factor (bFGF), which was also reversed by miR-126 inhibitor transfection. In conclusion, the present study demonstrated that miR-126 acts as a tumor suppressor in OSCC cells, partially at least via the downregulation of EGFL7. Thus, miR-126 may serve as a promising candidate for the treatment of OSCC.
\end{abstract}

Correspondence to: Professor Tianyou Ling, Department of Stomatology, The Second Xiangya Hospital of Central South University, no. 139 Renmin Road, Changsha, Hunan 410011, P.R. China

E-mail: csulingtianyou@163.com

Key words: oral squamous cell carcinoma, microRNA-126, epidermal growth factor-like domain 7, tumor suppressor

\section{Introduction}

Squamous cell carcinoma (SCC) is the most common malignancy of the oral cavity accounting for at least $92.8 \%$ of all oral malignancies (1). Furthermore, it accounts for $3 \%$ of all malignant tumors in humans. Every year, $>500,000$ individuals are diagnosed with oral squamous cell carcinoma (OSCC) worldwide (2). Over the last decade, despite improved diagnostic and therapeutic strategies, the 5-year survival rate of OSCC remains poor (3). Thus, the development of effective diagnostic and therapeutic candidates is urgently required.

Vasculogenesis and angiogenesis are important for the formation of new blood vessels, which is crucial in the development and progression of malignant tumors (4). This process is tightly regulated by various factors, including epidermal growth factor-like domain 7 (EGFL7). EGFL7, also known as VE-statin, MEGF7 and Notch4-like protein or Zneu1, was initially reported to inhibit the migration of human aortic smooth muscle cells in vitro, indicating that it may participate in vessel maturation (5). Further investigation revealed that EGFL7 was an important tubulogenic factor in the process of vasculogenesis (6). MicroRNA-126 (miR-126) is an endothelial-specific miRNA located within intron 7 of EGFL7 and has recently been found to be involved in the process of blood vessel formation. MicroRNAs (miRNAs) represent a class of $\sim 22$ nucleotide long, non-coding RNAs, which have been identified as important suppressive regulators of gene expression by the inhibition of protein translation, and to a lesser extent by mRNA degradation. Previously, accumulating evidence revealed the role of miR-126 in multiple types of cancer. Generally, the expression levels of miR-126 were downregulated in cancer tissues, while its overexpression significantly inhibited tumor growth, migration and invasiveness, partly through suppressing cancer cell proliferation. However, the role of miR-126 as well as the exact regulatory mechanism in OSCC remains to be fully elucidated.

In the present study, we examined the expression level of miR-126 as well as EGFL7 in 20 OSCC tissues, their matched adjacent tissues, as well as three OSCC cell lines, Tca8113, OSCC-15 and CAL27. Furthermore, an miR-126 mimic and miR-126 inhibitor were used to examine the role of miR-126 in OSCC-15 cells by examining cell proliferation, apoptosis, cell cycle distribution, colony formation and invasion. Furthermore, we also determined the effect of miR-126 overexpression and inhibition on the secretion of vascular endothelial growth 
factor (VEGF) and basic fibroblast growth factor (bFGF), respectively.

In conclusion, our data suggests that miR-126 acts as a tumor suppressor in OSCC cells and thus may serve as a promising diagnostic and therapeutic target for OSCC.

\section{Materials and methods}

Reagents and materials. Fetal bovine serum (FBS), TRIzol, TaqMan qRT-PCR miRNA assay kit, SYBR-Green qPCR mix, Lipofectamine 2000, miR-126 mimics and an miR-126 inhibitor were purchased from Thermo Fisher Scientific (Waltham, MA, USA). High glucose Dulbecco's modified Eagle's medium (H-DMEM) was purchased from Gibco Laboratories (Grand Island, NY, USA). Mouse anti-EGFL7 monoclonal antibody, mouse anti-GAPDH monoclonal antibody and rabbit anti-mouse secondary antibody were purchased from Abcam (Cambridge, UK). MTT was purchased from Sigma (St. Louis, MO, USA). Propidium iodide (PI) was purchased from Roche Molecular Biochemicals (Indianapolis, IN, USA). The cell invasion assay kit was obtained from Corning Inc. (Corning, NY, USA). The VEGF ELISA kit and bFGF ELISA kit were purchased from R\&D Systems (Minneapolis, MN, USA).

Tissue specimen collection. The present study was approved by the Ethics Committee of Xiangya Medical College, Central South University (Changsha, Hunan, China). Following informed consent being obtained from each patient, 10 cases of fresh-frozen OSCC tissues and their matched normal adjacent tissues, as well as 10 cases of normal tissues were obtained from patients at the Department of Stomatology, The Second Affiliated Xiangya Hospital of Central South University (Changsha, Hunan, China) from January to June 2012. No patient had undergone radiotherapy or chemotherapy prior to surgery. Following surgical removal, all samples were immediately snap-frozen in liquid nitrogen and stored at $-80^{\circ} \mathrm{C}$ until use.

Cell culture. Three OSCC cell lines, Tca8113, OSCC-15 and CAL27, were purchased from the American Type Culture Collection (ATCC; Manassas, VA, USA). All cells were cultured in DMEM supplemented with 10\% FBS, $100 \mathrm{IU} / \mathrm{ml}$ of penicillin and $100 \mu \mathrm{g} / \mathrm{ml}$ of streptomycin sulfate at $37^{\circ} \mathrm{C}$ in a humidified incubator containing $5 \% \mathrm{CO}_{2}$. All experiments were performed at the third passage.

RNA extraction and reverse transcription-polymerase chain reaction $(R T-P C R)$. Total RNA was extracted from tissues and cells using TRIzol. RNA was synthesized to cDNA using the RT-PCR kit in accordance with the manufacturer's instructions. For the EGFL7 assay, SYBR-Green qPCR mix was used to perform qRT-PCR. The EGFL7 primer was as follows: forward 5'-TGAATGCAGTGCTAGGAGGG-3' and reverse 5'-GCACACAGAGTGTACCGTCT-3'. Glyceraldehyde phosphate dehydrogenase (GAPDH) was used as an internal control with the sense primer 5'-ACAACTTTGGTATCGTGGAAGG-3' and antisense primer 5'-GCCATCACGCCACAGTTTC-3'. For the detection of miR-126 expression, the TaqMan qRT-PCR miRNA assay kit was used to perform real-time RT-PCR and analyzed with an ABI 7500 Sequence Detection System. U6 was used as an internal control. The relative expression levels of genes were analyzed using the $2^{-\Delta \Delta \mathrm{Ct}}$ method. Independent experiments were repeated three times.

Western blotting. Tissues or cells were solubilized in cold RIPA lysis buffer. Protein (20 $\mu \mathrm{g}$ per lane) was separated with $12 \%$ SDS-PAGE. Following that, the proteins were transferred onto nitrocellulose membranes, which were then inhibited in 5\% non-fat dried milk in PBST for $3 \mathrm{~h}$ and then incubated overnight with mouse anti-EFGL7 monoclonal antibody (1:200) or mouse anti-GAPDH monoclonal antibody (1:400). Following washing with PBS three times (each for $5 \mathrm{~min}$ ), the membranes were incubated with rabbit anti-mouse secondary antibody $(1: 40,000)$ for $1 \mathrm{~h}$ at room temperature. Then, the ECL kit (Huyu Co., Shanghai, China) was used to detect the immune complexes. Following that, the membranes were scanned for the relative value of protein expression in gray scale by Image-Pro plus software 6.0. The relative expression level of protein is presented as the density ratio versus GAPDH.

Transfection. Cells were cultured to $\sim 70 \%$ confluence and then were resuspended in serum-free H-DMEM at a concentration of 100,000 cells $/ \mathrm{ml}$. Six-well plates were used to inoculate with $2 \mathrm{ml}$ suspension for each well. According to the manufacturer's instructions, mir-126 mimics, NC mimics or an miR-126 inhibitor was diluted with $250 \mu \mathrm{l}$ of serum-free H-DMEM. Lipofectamine 2000 transfection reagent $(50 \mu \mathrm{l})$ was diluted with $2.5 \mathrm{ml}$ of serum-free H-DMEM. Then, the diluted Lipofectamine 2000 transfection reagent was added into the mimics or inhibitor dilution, mixed gently and incubated for $20 \mathrm{~min}$ at room temperature. The cell suspension was washed with serum-free H-DMEM two times, added with the mixture and then incubated at $37^{\circ} \mathrm{C}$ and $5 \% \mathrm{CO}_{2}$ for $6 \mathrm{~h}$. Following that, the medium in each well was replaced by the normal serum-containing medium and cultured for $24 \mathrm{~h}$ prior to the following experiments.

Cell proliferation assay. For each group, 10,000 cells per well were plated in a 96 -well plate. Then, the plates were incubated for $12,24,48$ or $72 \mathrm{~h}$ at $37^{\circ} \mathrm{C}$ and $5 \% \mathrm{CO}_{2}$. The MTT assay was performed to determine the cell proliferation. Each well was added with $25 \mu 1$ of MTT $(10 \mathrm{mg} / \mathrm{ml})$ and then incubated for $4 \mathrm{~h}$ at $37^{\circ} \mathrm{C}$ and $5 \% \mathrm{CO}_{2}$. Then, the supernatant was removed and each well was added with $150 \mu \mathrm{l}$ of DMSO. The absorbance was detected at $570 \mathrm{~nm}$ with a Microplate Reader (Bio-Rad, Hercules, CA, USA). Each assay was performed in triplicate wells.

Cell cycle distribution assay. At $48 \mathrm{~h}$ after transfection, cells were fixed with $70 \%$ ethanol. Then, the cells were stained with $25 \mathrm{lg} / \mathrm{ml}$ of PI in PBS containing $0.1 \%$ BSA, $0.05 \%$ Triton X-100 and $50 \mathrm{lg} / \mathrm{ml}$ of RNaseA for $30 \mathrm{~min}$ at room temperature. Following that, the cells were analyzed by flow cytometry.

Colony formation assay. For each group, each well of a 6-well plate was added with $3 \mathrm{ml}$ of complete medium containing 150 cells and then incubated at $37^{\circ} \mathrm{C}$ and $5 \% \mathrm{CO}_{2}$ for 14 days. Then, cells were gently washed and stained with Giemsa. 
Colonies containing at least 50 cells were counted and images were captured.

Cell invasion assay. The cell invasion assays were performed in a 24-well transwell chamber pre-coated with Matrigel. Cells were collected and resuspended in serum-free H-DMEM at a concentration of 10,000 cells $/ \mathrm{ml}$, respectively. The upper chamber was added with $0.2 \mathrm{ml}$ cell suspensions. The bottom chamber was filled with $0.5 \mathrm{ml}$ of H-DMEM containing $10 \%$ FBS. Following incubation for $24 \mathrm{~h}$ at $37^{\circ} \mathrm{C}$ and $5 \% \mathrm{CO}_{2}$, a cotton bud was used to remove the cells which were not through the polycarbonate membrane. Then, the cells which moved through the polycarbonate membrane and adhered to the bottom of it, were stained with Trypan blue for $15 \mathrm{~min}$, and then images were captured and the cells were counted.

Enzyme-linked immunosorbent assay (ELISA). Cell supernatants in each group were used to determine the secretion of VEGF and bFGF using ELISA. The VEGF and bFGF ELISA kits were used, according to the manufacturer's instructions, and the concentrations of VEGF and bFGF were calculated. Optical density (OD) values were determined by a microplate reader (Bio-Rad).

Statistical analysis. All data are expressed as the mean \pm SD of three independent experiments. Statistical analysis was performed using SPSS.17 software. Statistical analysis of differences was performed by one-way analysis of variance (ANOVA). $\mathrm{P}<0.05$ was considered to indicate a statistically significant difference $\left({ }^{* *} \mathrm{P}<0.05,{ }^{*} \mathrm{P}<0.01\right)$.

\section{Results}

Downregulation of miR-126 expression in OSCC tissues and cell lines. Firstly, we examined the expression level of miR-126 in 10 cases of OSCC samples and their matched adjacent tissues by performing real-time RT-PCR. As shown in Fig. 1A, the expression of miR-126 was significantly downregulated in OSCC tissues when compared with that in the matched normal tissues. We further determined the miR-126 expression level in three OSCC cell lines, Tca8113, OSCC-15 and CAL27. Our data demonstrated that the expression of miR-126 was also downregulated in three OSCC cell lines compared with the normal adjacent tissues (Fig. 1B). These findings indicate that miR-126 downregulation may be involved in the development of OSCC.

Upregulation of EGFL7 expression in OSCC tissues and cell lines. We further examined the protein level of EGFL7 in 10 cases of OSCC tissues and their matched adjacent tissues, as well as 10 cases of normal tissues. Western blot analysis data demonstrated that the expression of EGFL7 was notably increased in OSCC tissues compared with their matched adjacent and normal tissues (Fig. 2A). We also determined the EGFL7 protein level in Tca8113, OSCC-15 and CAL27 cells. As shown in Fig. 2B, all three cell lines demonstrated a positive protein expression of EGFL7.

miR-126 negatively regulates the protein expression of EGFL7 in OSCC-15 cells. Since bioinformatical analysis data
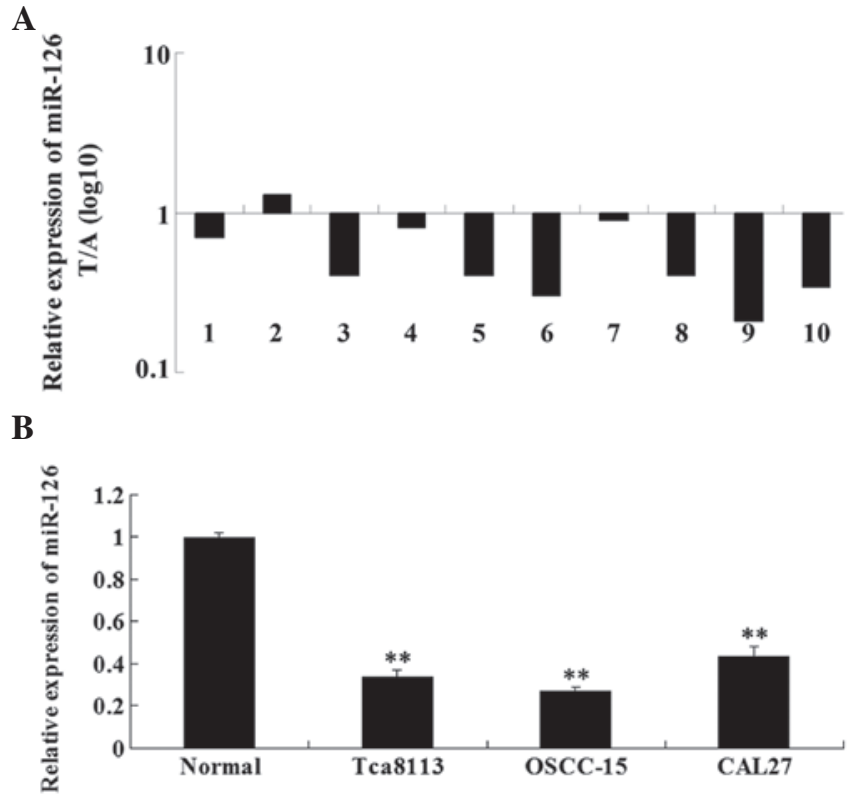

Figure 1. (A) Real-time RT-PCR was performed to determine the miR126 level in 10 OSCC patient samples and their matched adjacent tissues. (B) Real-time RT-PCR was performed to determine the miR-126 level in three OSCC cell lines, Tca8113, OSCC-15 and CAL27, as well as in normal tissues. ${ }^{* *} \mathrm{P}<0.01$ vs. normal. RT-PCR, reverse transcription-polymerase chain reaction; miR-126, microRNA-126; OSCC, oral squamous cell carcinoma.

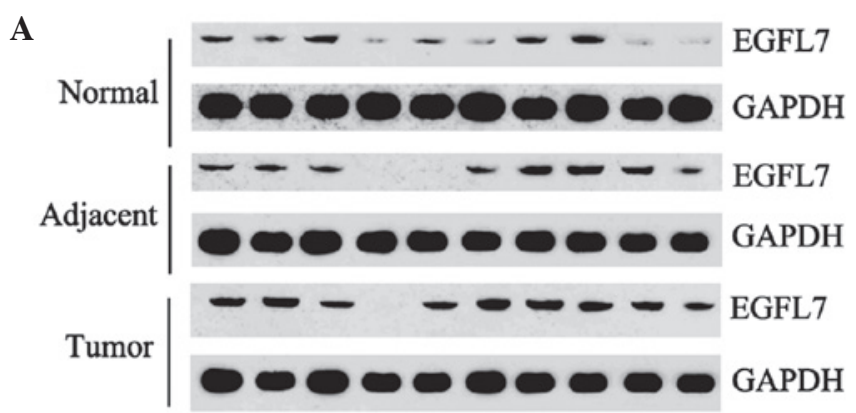

B

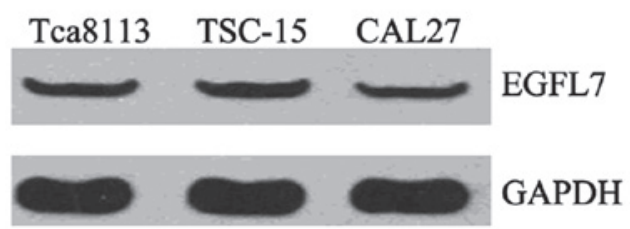

Figure 2. (A) Western blot analysis was performed to examine the protein expression of EGFL7 in 10 OSCC patient samples and their matched adjacent tissues, as well as 10 cases of normal tissues. GAPDH was used as an endogenous reference. (B) Western blot analysis was performed to examine the protein expression of EGFL7 in three OSCC cell lines, Tca8113, OSCC-15 and CAL27. GAPDH was used as an endogenous reference. EGFL7, epidermal growth factor-like domain 7; OSCC, oral squamous cell carcinoma; GAPDH, glyceraldehyde phosphate dehydrogenase.

demonstrated that EGFL7 was a putative target of miR-126, we preliminarily investigated their relationship in OSCC-15 cells. Following transfection with the miR-126 mimic and miR-126 inhibitor into OSCC-15 cells, respectively, real-time PCR was performed to determine the transfection efficiency. As shown in Fig. 3A, the expression level of miR-126 was markedly upregulated following transfection with the miR-126 


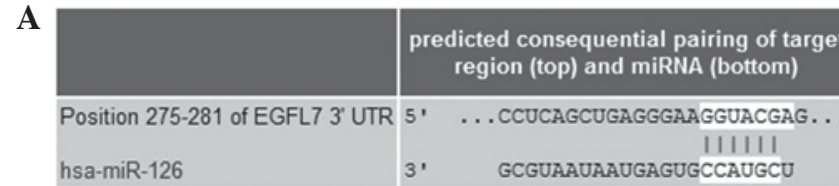

B
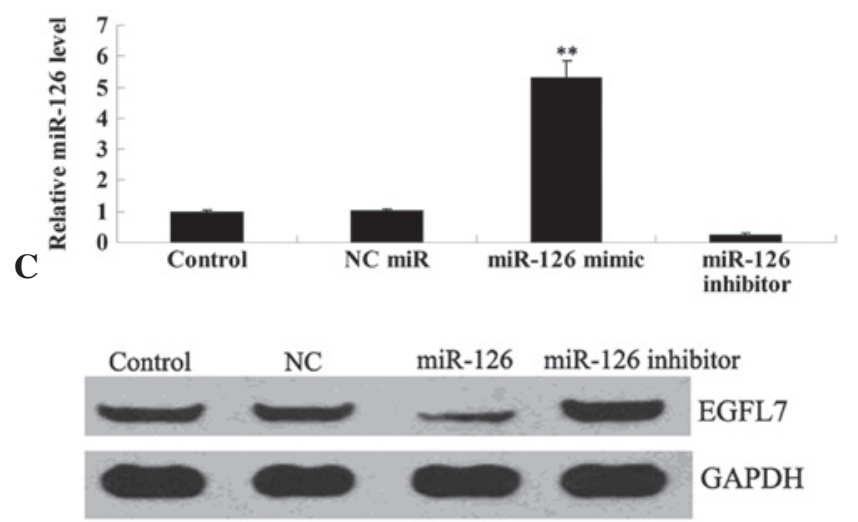

Figure 3. (A) TargetScan software demonstrated the putative target sequence of miR-126 in the 3'-UTR of EGFL7. (B) Real-time RT-PCR was performed to determine the miR-126 level following transfection of OSCC-15 cells with the NC miRNA, miR-126 mimic or miR-126 inhibitor. Control: cells without any transfection. NC miR: cells transfected with $\mathrm{NC}$ miRNA. ${ }^{* *} \mathrm{P}<0.01$ vs. control. (C) Western blot analysis was performed to examine the protein expression of EGFL7 following transfection of OSCC-15 cells with the NC miRNA, miR-126 mimic or miR-126 inhibitor. Control: cells without any transfection. EGFL7, epidermal growth factor-like domain 7; OSCC, oral squamous cell carcinoma; miR-126, microRNA-126; RT-PCR, reverse transcription-polymerase chain reaction; NC, negative control; GAPDH, glyceraldehyde phosphate dehydrogenase; miRNA, microRNA.

mimic, and notably downregulated following transfection with the miR-126 inhibitor, when compared with that in the control group, respectively. Following confirmation of the transfection efficiency, we applied western blotting to examine the protein expression of EGFL7 in each group. As demonstrated in Fig. 3B, the expression level of EGFL7 was reduced following transfection with the miR-126 mimic in OSCC-15 cells. By contrast, the EGFL7 expression level was upregulated in OSCC-15 cells transfected with the miR-126 inhibitor. These findings suggest that miR-126 negatively regulates the protein expression of EGFL7 in OSCC-15 cells.

Effects of miR-126 upregulation and downregulation on OSCC-15 cell proliferation. We further determined the effects of miR-126 overexpression or inhibition on OSCC-15 cell proliferation. As shown in Fig. 4, the overexpression of miR-126 significantly downregulated the cellular proliferation of OSCC-15 cells, while the downregulation of miR-126 by its inhibitor markedly upregulated OSCC-15 cell proliferation. Accordingly, we suggested that miR-126 has an inhibitory effect on OSCC-15 cell proliferation.

Role of miR-126 in OSCC-15 cell apoptosis. We investigated the role of miR-126 in OSCC-15 cell apoptosis. As demonstrated in Fig. 5, the transfection of OSCC-15 cells with the miR-126 mimic significantly upregulated their apoptosis, which could be reversed by transfection with the miR-126 inhibitor. Thus, we suggested that miR-126 can induce cell apoptosis in OSCC-15 cells.

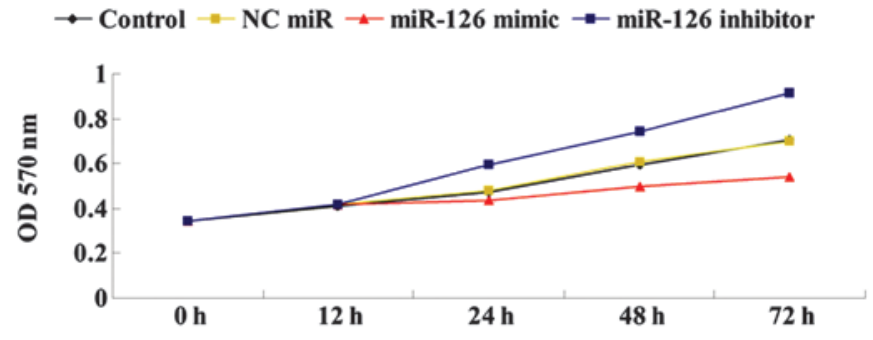

Figure 4. Cell proliferation assay was performed following transfection of OSCC-15 cells with the NC miRNA, miR-126 mimic or miR-126 inhibitor. Control: cells without any transfection. $\mathrm{NC}$ miR: cells transfected with $\mathrm{NC}$ miRNA. miR-126, microRNA-126; NC, negative control; OSCC, oral squamous cell carcinoma; OD, optical density; miRNA, microRNA.
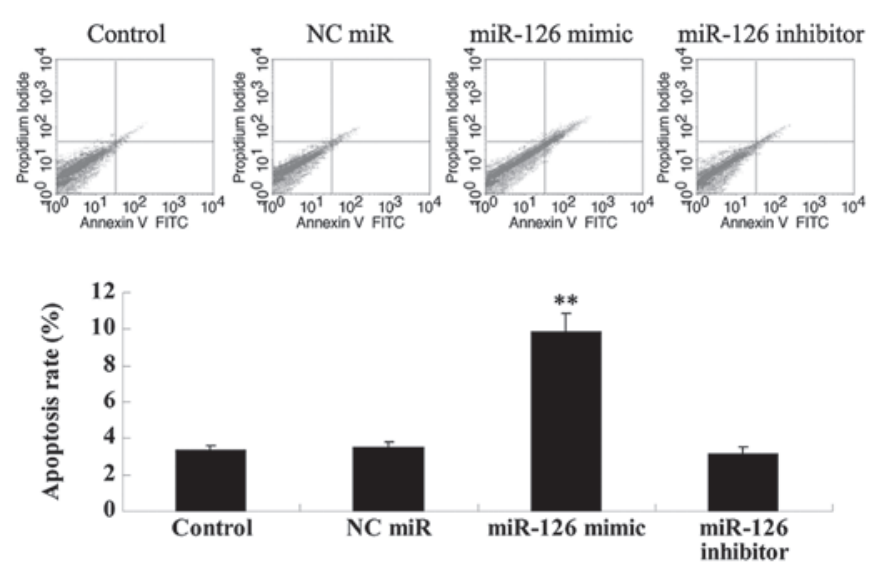

Figure 5. Cell apoptosis assay was performed to determine the apoptosis rate following transfection of OSCC-15 cells with the NC miRNA, miR-126 mimic or miR-126 inhibitor. Control: cells without any transfection. NC miR: cells transfected with NC miRNA. ${ }^{* *} \mathrm{P}<0.01$ vs. control. miR-126, microRNA-126; NC, negative control; OSCC, oral squamous cell carcinoma; miRNA, microRNA.

Effect of miR-126 on the cell cycle progression of OSCC-15 cells. To further investigate the effect of miR-126 on the cell cycle progression of OSCC-15 cells, a cell cycle assay was performed. Our findings demonstrated that the miR-126 overexpressed OCSS-15 cells demonstrated the highest percentage in the G1 stage, however, the lowest percentage in the S stage, when compared with that in other groups, indicating that mitosis was inhibited in the G1 stage. By contrast, OCSS-15 cells transfected with the miR-126 inhibitor demonstrated the highest percentage in the G2/M stage, indicating that the inhibition of miR-126 promoted mitosis (Fig. 6). These findings suggest that miR-126 has a negative effect on cell cycle progression in OCSS-15 cells.

Role of miR-126 in the regulation of cell invasion in OSCC-15 cells. The cell invasion ability of OSCC-15 cells was examined following transfection with the miR-126 mimic and inhibitor, respectively. As shown in Fig. 7, miR-126 overexpression markedly inhibited OSCC-15 cell invasion, while miR-126 inhibition significantly promoted the invasion of OSCC-15 cells, when compared with that in the control groups.

Effects of miR-126 upregulation and inhibition on the VEGF and bFGF secretion in OSCC-15 cells. As EGFL7 has been demonstrated to be implicated in the regulation of angiogenesis, 

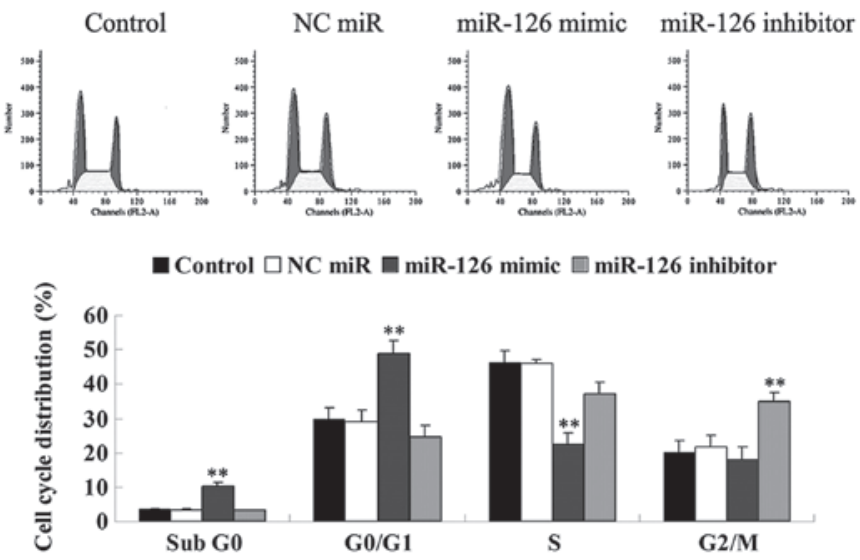

Figure 6. Cell cycle assay was performed to investigate the cell cycle distribution following transfection of OSCC-15 cells with the NC miRNA, miR-126 mimic or miR-126 inhibitor. Control: cells without any transfection. NC miR: cells transfected with NC miRNA. ${ }^{* *} \mathrm{P}<0.01$ vs. control. NC, negative control; miR-126, microRNA-126; OSCC, oral squamous cell carcinoma; miRNA, microRNA.
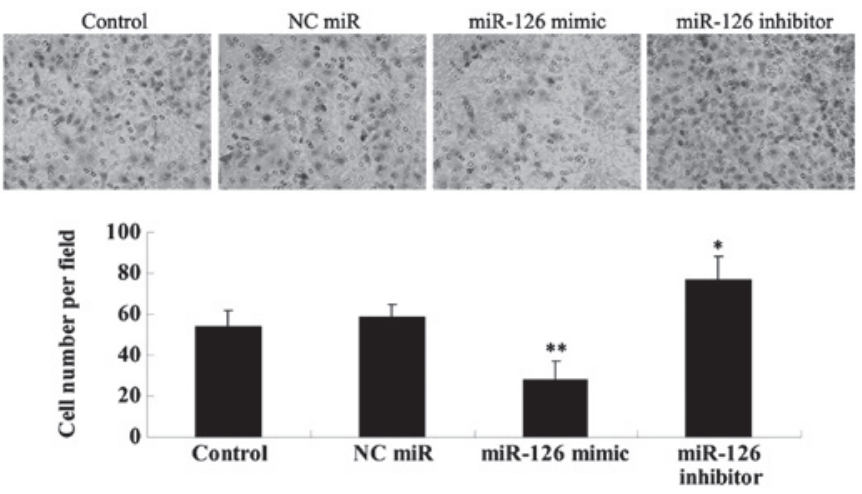

Figure 7. Cell invasion assay was performed to examine the cell invasion ability following transfection of OSCC-15 cells with the NC miRNA, miR-126 mimic or miR-126 inhibitor. Five fields were randomly selected and the cell number was counted. Control: cells without any transfection. NC miR: cells transfected with $\mathrm{NC}$ miRNA. ${ }^{*} \mathrm{P}<0.05$ vs. control. ${ }^{* *} \mathrm{P}<0.01$ vs. control. NC, negative control; miR-126, microRNA-126; OSCC, oral squamous cell carcinoma; miRNA, microRNA.

we performed ELISA to determine the secretion levels of two vascular markers VEGF and bFGF in OSCC-15 cells transfected with the miR-126 mimic and inhibitor, respectively. Our findings demonstrated that miR-126 overexpression significantly downregulated the secretion of VEGF and bFGF in OSCC-15 cells, while miR-126 inhibition markedly promoted their secretion (Fig. 8). These findings suggest that miR-126 may possess an inhibitory function in angiogenesis, partially at least via suppressing EGFL7 expression.

Effects of miR-126 upregulation and downregulation on the colony-formation efficiency of OSCC-15 cells. The effects of miR-126 overexpression and inhibition on colony-formation efficiency in OSCC-15 cells were investigated. As demonstrated in Fig. 9, the miR-126 overexpressed OSCC-15 cells demonstrated the lowest colony-formation efficiency, while the miR-126 downregulated OSCC-15 cells demonstrated the highest colony-formation efficiency, when compared with that in the controls.
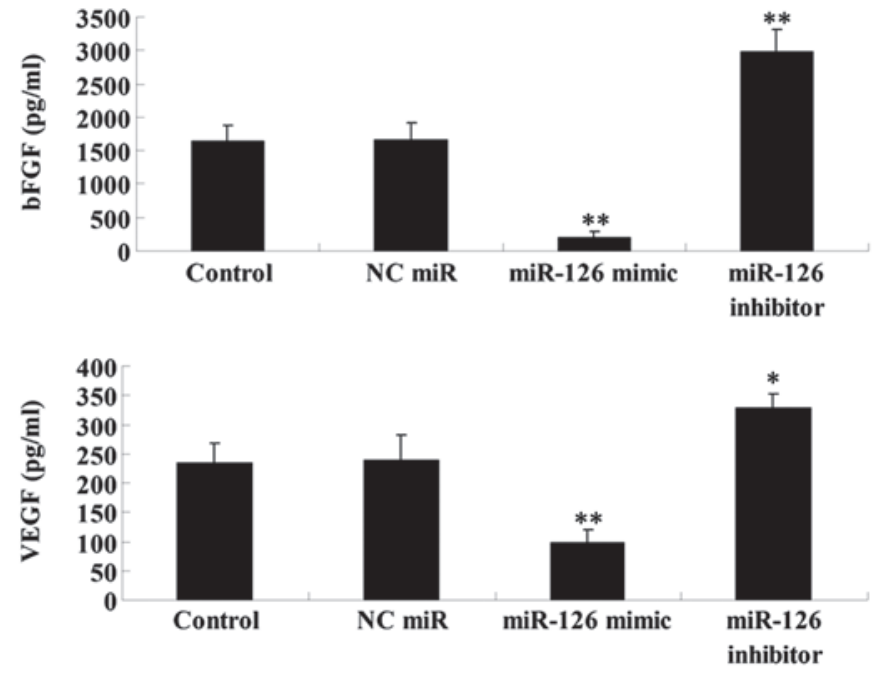

Figure 8. ELISA was performed to examine the secretion level of bFGF and VEGF following transfection of OSCC-15 cells with NC miRNA, miR-126 mimic or miR-126 inhibitor. Control: cells without any transfection. NC miR: cells transfected with NC miRNA. ${ }^{*} \mathrm{P}<0.05$ vs. control. ${ }^{* *} \mathrm{P}<0.01$ vs. control. ELISA, enzyme-linked immunosorbent assay; NC, negative control; OSCC, oral squamous cell carcinoma; miR-126, microRNA-126; VEGF, vascular endothelial growth factor; bFGF, basic fibroblast growth factor; miRNA, microRNA.

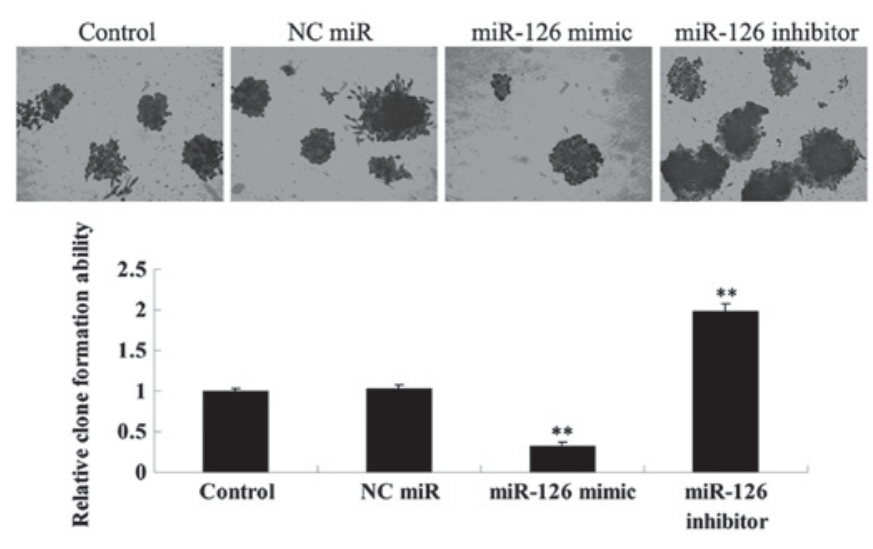

Figure 9. Colony formation assay was performed to examine the colony formation ability following transfection of OSCC-15 cells with the NC miRNA, miR-126 mimic or miR-126 inhibitor. Control: cells without any transfection. NC miR: cells transfected with NC miRNA. ${ }^{* *} \mathrm{P}<0.01$ vs. control. NC, negative control; miR-126, microRNA-126; OSCC, oral squamous cell carcinoma; miRNA, microRNA.

\section{Discussion}

miR-126 has been revealed to be important in different physiological and pathological processes, including inflammation (7), blood vessel formation (8) as well as the development and progression of malignant tumors (9). Altered expression of miR-126 has been reported in various cancer cells, including breast cancer, colon cancer, prostate cancer, lung cancer, cervical cancer, bladder cancer, prostate cancer, gastric cancer and leukemia (9-16). However, the function of miR-126 in OSCC cells remains largely unknown. The present study suggests that miR-126 has a suppressive effect on OSCC cells, mainly through inhibiting cell proliferation, cell cycle progression, cell invasion and colony formation, as well as inducing 
cell apoptosis. Furthermore, miR-126 upregulation reduced VEGF and bFGF secretion, which are critical drivers for angiogenesis and subsequent tumor growth (17). These findings indicate that miR-126 acts as a tumor suppressor in OSCC cells.

Recently, an increasing body of evidence has focused on the role of miR-126 in various types of cancer. Guo et al demonstrated that miR-126 inhibited colon cancer possibly via the phosphatidylinositol 3-kinase signaling pathway (18). Liu et al reported that miR-126 overexpression induced cell cycle arrest in vitro and inhibited tumor cell growth in vivo, partially through negatively targeting VEGF-A in lung carcinoma cells (19). In fact, the expression level of miR-126 was also regulated by oncogenes. For instance, Li et al reported that overexpression of the oncogene Src caused a decrease in miR-126 expression in mouse embryonic $\mathrm{Cx} 43 \mathrm{KO}$ brain cells and the Src kinase inhibitor PP2 led to an increase in miR-126 expression, which further decreased cell migration in breast cancer cells (20). Furthermore, miR-126 was found to directly target the 3'-UTR of Crk, the downregulation of which leads to the inhibition of tumor cell proliferation and invasion, through affecting the focal adhesion network involved in integrin signaling $(16,21)$. Accordingly, miR-126 has been demonstrated to be involved in multiple molecular signaling cascades in various cancer cells.

Furthermore, miR-126 is an intron-located miRNA and its host gene is EGFL7. Generally, the expression of the majority of the intron-located miRNA is paralleled by the transcription of mRNAs encoded by the host genes. Recently, however, certain regulatory elements upstream of the host genes were identified to promote intron-located miRNA transcription independently. Thus, an independent expression of miRNAs and mRNAs may also exist. Indeed, one study previously reported that the expression of miR-126 was regulated independently of EGFL7 (20), indicating that a separate promoter may exist, driving miR-126 transcription. However, whether or not this promoter element exists remains to be elucidated by further investigations. In the present study, we demonstrated that the expression of miR-126 negatively regulated the protein level of EGFL7 and bioinformatical analysis data also demonstrated that EGFL7 was a direct target of miR-126. These findings suggest that although the expression pattern of miR-126 and EGFL7 remains controversial, miR-126 is likely to inhibit EGFL7 expression at a post-transcriptional level.

Furthermore, several studies have indicated that miR-126 can promote endothelial cell proliferation and migration $(8,22)$ and thus may be essential for angiogenesis. In theory, this putative function is counterintuitive for its suppressive role in various types of cancer. However, the exact role of miR-126 in tumor angiogenesis remains largely uncovered. To connect the role of miR-126 in cancer cells to its physiological function in the vasculature, we examined the effect of miR-126 upregulation and downregulation on the secretion of VEGF and bFGF, two key regulators for angiogenesis as well as cancer development, and found that miR-126 had an inhibitory effect on their production. Since blood vessel growth represents a key feature in the pathogenesis of cancer, further investigation regarding the effect of miR-126 on the tumor vasculature and the surrounding vessels can help reveal its exact role in cancer progression.

In summary, the present study suggests that miR-126 has a suppressive effect in OSCC cells and thus may become a promising candidate for the development of a therapeutic strategy for OSCC treatment.

\section{References}

1. Lo Muzio L, Santarelli A, Panzarella V, et al: Oral squamous cell carcinoma and biological markers: an update on the molecules mainly involved in oral carcinogenesis. Minerva Stomatol 56: 341-347, 2007.

2. Fan S, Tang QL, Lin YJ, et al: A review of clinical and histological parameters associated with contralateral neck metastases in oral squamous cell carcinoma. Int J Oral Sci 3: 180-191, 2011.

3. Shi Z and Stack MS: Molecules of cell adhesion and extracellular matrix proteolysis in oral squamous cell carcinoma. Histol Histopathol 25: 917-932, 2010.

4. Albini A, Tosetti F, Li VW, Noonan DM and Li WW: Cancer prevention by targeting angiogenesis. Nat Rev Clin Oncol 9: 498-509, 2012.

5. Soncin F, Mattot V, Lionneton F, et al: VE-statin, an endothelial repressor of smooth muscle cell migration. EMBO J 22: 5700-5711, 2003.

6. Parker LH, Schmidt M, Jin SW, et al: The endothelial-cell-derived secreted factor Egfl7 regulates vascular tube formation. Nature 428: 754-758, 2004.

7. Harris TA, Yamakuchi M, Ferlito M, Mendell JT and Lowenstein CJ: MicroRNA-126 regulates endothelial expression of vascular cell adhesion molecule 1. Proc Natl Acad Sci USA 105: 1516-1521, 2008.

8. Wang S, Aurora AB, Johnson BA, et al: The endothelial-specific microRNA miR-126 governs vascular integrity and angiogenesis. Dev Cell 15: 261-271, 2008.

9. Meister J and Schmidt MH: miR-126 and miR-126*: new players in cancer. ScientificWorldJournal 10: 2090-2100, 2010.

10. Wang X, Tang S, Le SY, et al: Aberrant expression of oncogenic and tumor-suppressive microRNAs in cervical cancer is required for cancer cell growth. PLoS One 3: e2557, 2008.

11. Crawford M, Brawner E, Batte K, et al: MicroRNA-126 inhibits invasion in non-small cell lung carcinoma cell lines. Biochem Biophys Res Commun 373: 607-612, 2008.

12. Ladeiro Y, Couchy G, Balabaud C, et al: MicroRNA profiling in hepatocellular tumors is associated with clinical features and oncogene/tumor suppressor gene mutations. Hepatology 47: 1955-1963, 2008

13. Li Z, Lu J, Sun M, et al: Distinct microRNA expression profiles in acute myeloid leukemia with common translocations. Proc Natl Acad Sci USA 105: 15535-15540, 2008.

14. Saito Y, Friedman JM, Chihara Y, Egger G, Chuang JC and Liang G: Epigenetic therapy upregulates the tumor suppressor microRNA-126 and its host gene EGFL7 in human cancer cells. Biochem Biophys Res Commun 379: 726-731, 2009.

15. Musiyenko A, Bitko V and Barik S: Ectopic expression of miR-126*, an intronic product of the vascular endothelial EGF-like 7 gene, regulates prostein translation and invasiveness of prostate cancer LNCaP cells. J Mol Med (Berl) 86: 313-322, 2008.

16. Feng R, Chen X, Yu Y, et al: miR-126 functions as a tumour suppressor in human gastric cancer. Cancer Lett 298: 50-63, 2010.

17. Bremnes RM, Camps C and Sirera R: Angiogenesis in non-small cell lung cancer: the prognostic impact of neoangiogenesis and the cytokines VEGF and bFGF in tumours and blood. Lung Cancer 51: 143-158, 2006.

18. Guo C, Sah JF, Beard L, Willson JK, Markowitz SD and Guda K: The noncoding RNA, miR-126, suppresses the growth of neoplastic cells by targeting phosphatidylinositol 3-kinase signaling and is frequently lost in colon cancers. Genes Chromosomes Cancer 47: 939-946, 2008.

19. Liu B, Peng XC, Zheng XL, Wang J and Qin YW: MiR-126 restoration down-regulate VEGF and inhibit the growth of lung cancer cell lines in vitro and in vivo. Lung Cancer 66: 169-175, 2009.

20. Li X, Shen Y, Ichikawa H, Antes T and Goldberg GS: Regulation of miRNA expression by Src and contact normalization: effects on nonanchored cell growth and migration. Oncogene 28: 4272-4283, 2009.

21. Birge RB, Kalodimos C, Inagaki F and Tanaka S: Crk and CrkL adaptor proteins: networks for physiological and pathological signaling. Cell Commun Signal 7: 13, 2009.

22. Fish JE, Santoro MM, Morton SU, et al: miR-126 regulates angiogenic signaling and vascular integrity. Dev Cell 15: 272-284, 2008 . 